\title{
Estado de conservación de Lagothrix flavicauda y otros primates en bosques montanos de San Martín y Huánuco, Perú
}

\section{Conservation status of Lagothrix flavicauda and other primates in montane forest of San Martín and Huánuco, Peru}

\section{Rolando Aquino ${ }^{1}$, Gabriel García ${ }^{2}$, Elvis Charpentier ${ }^{2}$ y Luís López ${ }^{3}$}

\author{
1 Universidad Nacional Mayor de San Marcos, Facultad de Ciencias Biológicas, Lima, Perú. \\ 2 Equipo Primatológico de Loreto, Iquitos, Perú. \\ 3 Universidad Nacional de la Amazonía Peruana, Iquitos, Perú \\ Email Rolando Aquino: raquinoy2005@yahoo.es \\ Email Gabriel García: garcia.mendoza25@gmail.com \\ Email Elvis Charpentier: primates2012@gmail.com \\ Email Luis López: luislopezramirez24@gmail.com
}

\section{Resumen}

Lagothrix flavicauda es endémica y vive en simpatría con otros primates en bosques montanos de Amazonas, San Martín, La Libertad y Huánuco. Información detallada sobre su abundancia y estado de conservación, así como de los sitios con poblaciones saludables son desconocidas, excepto para Amazonas donde fueron ejecutados la mayoría de los estudios. Para obtener la información sobre los aspectos mencionados fueron conducidos censos por transecto entre noviembre y diciembre del 2015, febrero a mayo del 2016 y entre junio y julio del 2016 en seis sitios de muestreo localizados entre las regiones de San Martín y Huánuco. Como resultado de los censos fueron avistados 49 grupos pertenecientes a 7 especies, siendo L. flavicauda la más observada con 14 grupos. Grupos más grandes se observaron en $L$. flavicauda (promedio 13.5 \pm 2.2 individuos) y Cebus yuracus (promedio $12.5 \pm 3.6$ individuos) y más pequeños en Alouatta seniculus (promedio $3.0 \pm 2.4$ individuos), ambos en Huánuco. La abundancia relativa fue más alta para $L$. flavicauda en San Martín (2.4 individuos $/ 10 \mathrm{~km}$ ) y más baja para $A$. seniculus en Huánuco $(0.2$ individuos $/ 10 \mathrm{~km})$. Con el registro de L. flavicauda en el lado oriental del río Huallaga su distribución en Huánuco se amplía hasta el río Pozuzo, pero en el lado occidental podemos considerarlo localmente extinta en gran parte de su distribución. En los sitios de muestreo, la deforestación y la caza son las principales amenazas para los primates y otros componentes de la fauna silvestre.

Palabras clave: Choro Cola Amarilla; Bosque montano andino; abundancia relativa; amenazas; deforestación; caza.

\section{Abstract}

Lagothrix flavicauda is endemic and lives sympatrically with other primates in the montane forests of Amazonas, San Martín, La Libertad and Huánuco. Detailed information on its density and conservation status, as well as sites with healthy populations is lacking, except for Amazonas where most of the studies were carried out. In order to obtain information on the mentioned aspects, transect censuses were conducted between November and December of 2015, February to May of 2016, and between June and July of 2016 in six survey sites located in the regions of San Martín and Huánuco. As a result of the census, 49 groups belonging to 7 species were sighted, being $L$. flavicauda the most observed with 14 groups. The largest groups were observed in $L$. flavicauda (average $13.5 \pm 2.2$ individuals) and Cebus yuracus (average 12.5 \pm 3.6 individuals) and the smallest in Alouatta seniculus (average $3.0 \pm 2.4$ individuals), both in Huánuco. The relative abundance was the highest for $L$. flavicauda in San Martín (2.4 individuals $/ 10 \mathrm{~km})$ and the lowest for A. seniculus in Huánuco (0.2 individuals/ $10 \mathrm{~km}$ ). Our observation of $L$. flavicauda on the eastern side of the Huallaga River extends its distribution in Huánuco to the Pozuzo River, but on the western side we can consider it locally extinct in much of its distributional range. At our survey sites, deforestation and hunting are the main threats to primates and other components of wildlife.

Keywords: Yellow-tailed Woolly Monkey; Andean montane forest; relative abundance; threats; deforestation; hunting

\section{Citación:}

Aquino R., G. García, E. Charpentier y L. López. 2017. Estado de conservación de Lagothrix flavicauda y otros primates en bosques montanos de San Martín y Huánuco, Perú. Revista peruana de biología 24(1): 025 - 034 (Abril 2017). doi: http://dx.doi.org/10.15381/rpb.v24i1.13101

Presentado: $\quad 12 / 12 / 2016$

Aceptado: $\quad 28 / 03 / 2017$

Publicado online: $20 / 04 / 2017$
Información sobre los autores:

RA realizó las coordinaciones con autoridades locales, registros de datos en campo, análisis y redacción del manuscrito. GG y EC realizaron los registros de campo y elaboró la base de datos y el mapa. LAL apoyó en el análisis de datos y redacción del manuscrito.

Los autores no incurren en conflictos de intereses.

Fuentes de financiamiento:

The Mohamed bin Zayed Species Conservation Fund (\# 152510817); Universidad Nacional Mayor de San Marcos-VRIP (\#161001211) 


\section{Introducción}

En la Amazonía peruana, los bosques montanos del centro y suroriente son los menos explorados, por lo que la información acerca de la diversidad y estado actual de los primates y otros componentes de la fauna silvestre que habitan en ellos es muy escasa. La mayoría de estudios sobre primates fueron conducidos en bosques montanos de la Región Amazonas y están referidos específicamente a Lagothrix flavicauda (Leo Luna 1980, 1982, 1987, Cornejo 2008, Shanee et al. 2008, Shanee 2011) y Aotus miconax (Butchart et al. 1995, Cornejo et al. 2008, Shanee et al. 2013a). A ellos se suman el inventario de primates en el interfluvio de los ríos Marañón-Huallaga (Shanee et al. 2013b) y bosques pre montano y montano de Cajamarca (Aquino et al. 2014). Para el resto, con excepción de los bosques montanos de Huánuco, prácticamente no existe información y se debe entre otros factores al difícil acceso por su relieve escarpado y accidentado, su lejanía de los centros poblados y las condiciones climáticas. Con respecto a los primates que habitan en bosques montanos de Huánuco, las únicas referencias son de Shanee et al. (2013b) que reportan cuatro especies, en tanto que Aquino \& Ramos (2010) y Aquino et al. (2015a), dan a conocer la existencia de seis especies. La escasa información acerca de L. flavicauda considerada hasta el 2012 entre las 25 especies más amenazadas del mundo (Mittermeier et al. 2012) y de otros primates que habitan en los bosques montanos de San Martín y Huánuco nos motivó a la conducción de este estudio, cuyos objetivos estuvieron orientados a: identificar las especies con quienes $L$. flavicauda comparte el hábitat, determinar la situación actual de sus poblaciones y del resto de especies e identificar sitios con poblaciones en buen estado de conservación para recomendar como áreas de Conservación y para el ecoturismo. Para cumplir con los objetivos propuestos fueron conducidos censos por tran- secto entre noviembre y diciembre del 2015 en bosques aledaños a San Antonio, de febrero hasta abril del 2016 en bosques de Tingo de Uchiza, Shunté y Libertad y de mayo a julio del 2016 en bosques de Chapákara y Hoja Grande. El propósito de los censos fue para determinar el tamaño de los grupos y abundancia y de paso constatar in situ el nivel de perturbación de los bosques por actividades antrópicas, aspectos fundamentales que influyen para la supervivencia de L. flavicauda y de los otros primates que habitan en el área de estudio. En este reporte se presentan los resultados obtenidos.

\section{Área de estudio}

De acuerdo con Young y León (1999), los bosques montanos de las regiones de San Martín y Huánuco pertenecen al denominado bosque montano oriental, ubicado en el lado amazónico de la Cordillera Oriental, sobre aproximadamente $1500 \mathrm{~m}$ de altitud y que recibe una amplia influencia de la Amazonia en cuanto a clima y biodiversidad. En estas regiones, los bosques están expuestos a una intensa deforestación para la agricultura, ganadería y extracción de recursos forestales, por lo que actualmente predominan los bosques secundarios y residuales altamente intervenidos, en tanto que fragmentos de bosque primario se encuentran mayormente en los relieves accidentados donde no es posible la agricultura ni la ganadería. La caza, a diferencia de la selva baja, es esporádica y orientada al consumo de subsistencia, venta (de infantes como mascotas y pieles para artesanía) y control de poblaciones, esta última practicada principalmente por colonos de origen andino para evitar pérdidas económicas de sus cultivos.

En el área, para los fines de inventario y evaluación fueron definidos seis sitios de muestreo (Fig. 1), cuya información acerca de su ubicación geográfica, altitud y nivel de perturbación

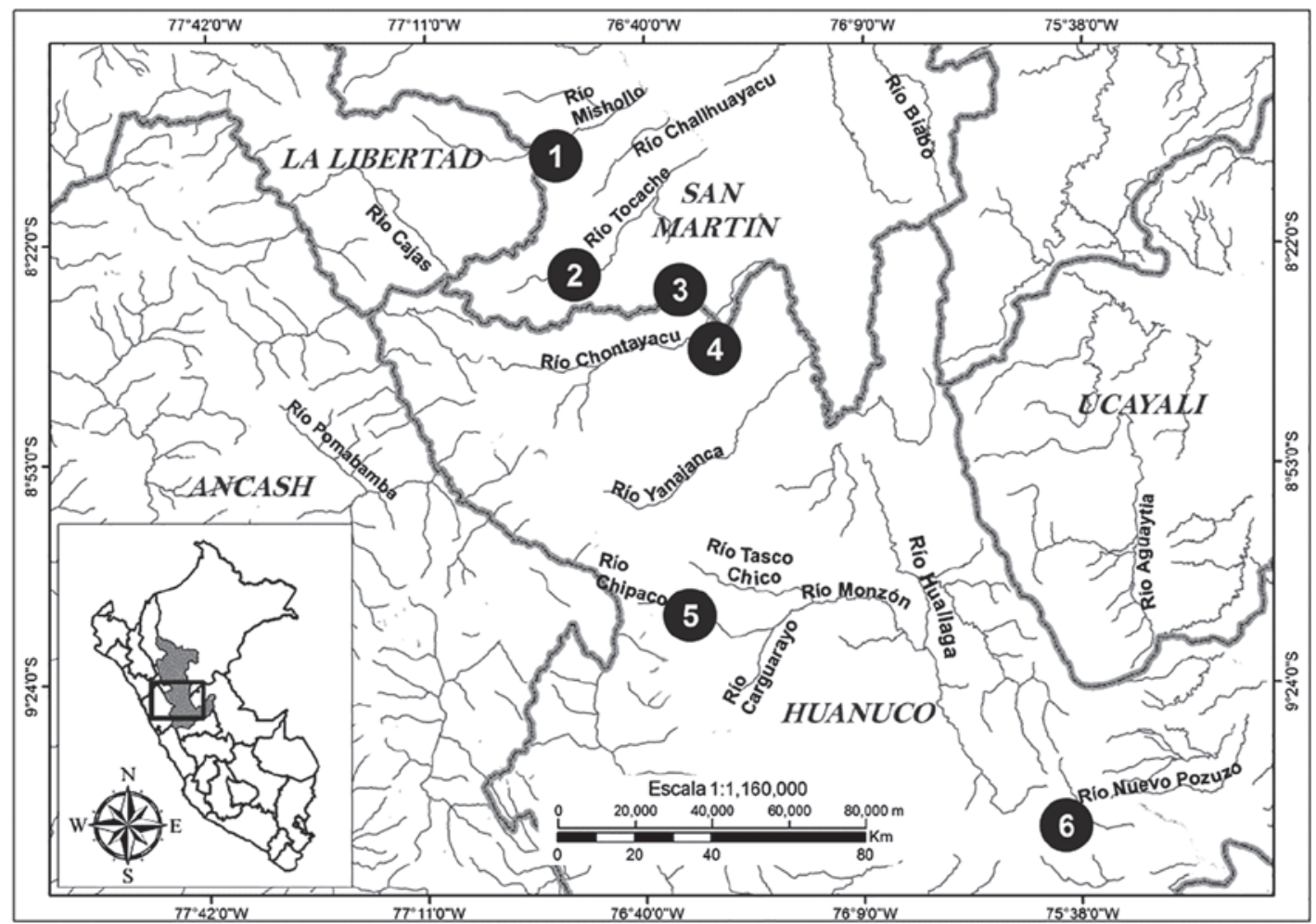

Figura 1. Sitios de muestreo por sectores para el inventario y evaluación de primates entre San Martín y Huánuco. Los números corresponden a la lista en la Tabla 1. 
humana se detalla en la Tabla 1. Para los fines de este estudio, se denomina nivel de muy alta perturbación humana a los bosques que presentan serias alteraciones por la extracción de madera y otros recursos forestales de modo que la altura promedio de los árboles no sobrepasa los $20 \mathrm{~m}$, salvo algunos emergentes arriba de $25 \mathrm{~m}$, y se debe a la ausencia o escasez de especies maderables. A ellos se suma la caza y la existencia de numerosas trochas, caminos y campamentos en uso para el desarrollo de las actividades antes mencionadas. Otra de las características a resaltar de estos bosques es la escasez de primates y otros componentes de la fauna mayor, quienes ante la presencia humana huyen despavoridos, lo que indica que están expuestos a la fuerte perturbación de su hábitat y la caza. El bosque de alta perturbación es similar al anterior; la diferencia está en la mayor presencia de primates y otros componentes de la fauna mayor, porque la caza es más específica y está orientada hacia los animales considerados como plaga para la agricultura, por lo que algunas especies de primates que no ingresan a los campos de cultivo, como es el caso de L. flavicauda y Ateles chamek, son todavía tolerantes a la presencia humana.

Los censos y exploraciones con fines de inventario y abundancia fueron conducidos mayormente en bosque residual esencialmente primario, es decir, bosque que aún conserva la estructura y la composición florística de un bosque primario no intervenido, ya que la extracción de madera como producto principal no lo ha modificado drásticamente. Los sitios de muestreo (Fig. 1) fueron los siguientes:

En la microcuenca del río Mishollo, el bosque aledaño a Hoja Grande en el límite con la Región La Libertad. En este sitio de muestreo predomina el bosque residual esencialmente primario con extensiones que superan los $112 \mathrm{~km}^{2}$, mientras que parches de bosque primario y remanente se encuentran en las laderas accidentadas. La deforestación para la agricultura y ganadería es moderada; sin embargo, el bosque muestra un perfil de alta perturbación por la extracción de árboles maderables y otros recursos forestales y la presión de caza que es relativamente alta.

En la microcuenca del río Tocache, el bosque aledaño al centro poblado de Shunté. Aquí, predomina el bosque residual esencialmente primario, cuyas extensiones superan los $15 \mathrm{~km}^{2}$, en tanto que la deforestación para los fines agrícolas y ganaderas se realizan mayormente aguas abajo del centro poblado. La caza es otra de las actividades practicadas y está orientada al consumo de subsistencia y obtención de infantes para su venta como mascotas.
En la microcuenca del río Crisnejas, el bosque cercano al centro poblado de Tingo de Uchiza. En este sitio de muestreo los fragmentos de bosque residual relativamente extensos (12 a $32 \mathrm{~km}^{2}$ ) están expuestos a la extracción de recursos forestales y a la caza con fines de subsistencia, mientras que la deforestación para la agricultura y ganadería es moderada debido a la predominancia de relieves sumamente accidentados.

En la microcuenca del río Chontayacu, el bosque aledaño al centro poblado de San Antonio. En esta parte, el bosque residual presente en ambas márgenes del río supera los $28 \mathrm{~km}^{2}$, pero está muy perturbado por la extracción de recursos forestales con fines comerciales y la caza de subsistencia, cuya presión es relativamente alta. Por otro lado, la deforestación para la agricultura todavía es moderada, aun cuando desde fines del 2012 se nota un incremento notable debido a la apertura de la carretera Uchiza - Huacrachuco, que está permitiendo el asentamiento de más colonos de procedencia andina.

En la microcuenca del río Monzón, el bosque cercano al centro poblado de Chapákara, asentado en la margen izquierda del río Yanamayo, afluente derecho del río Monzón. En esta parte del Monzón, los fragmentos de bosque residual que no superan los $4.5 \mathrm{~km}^{2}$ están expuestos a una constante intervención humana por la extracción de madera y otros recursos como lianas para la construcción de viviendas y corrales de animales. Otras actividades son la extracción de resina de Clusia sp. para el comercio como incienso y la caza con fines de subsistencia y venta de pieles.

En el interfluvio de los ríos Huallaga y Pozuzo, los bosques circundantes al Fundo Libertad y Oso Mayo, localizados aproximadamente a $15 \mathrm{~km}$ del centro poblado de Monopampa y a $20 \mathrm{~km}$ del río Huallaga. Al igual que el resto de sitios de muestreo, el bosque residual esencialmente primario está siendo deforestado para el cultivo de café, maíz y otros productos de pan llevar, así como para la ganadería. Sin embargo, todavía existen fragmentos de bosque relativamente extensos en buen estado de conservación, algunos mayores a $23 \mathrm{~km}^{2}$ donde se encuentran habitando primates y otros componentes de la fauna silvestre.

Adicionalmente, se llevaron a cabo exploraciones cortas en bosques cercanos a los centros poblados de César Vallejo y Fujimori asentados en la microcuenca del río Tulumayo y Herminio Valdizán, Ugartechi, Río Azul y Cedropampa, todos localizados cerca al límite con la Región Ucayali. El principal propósito de estas expediciones fue indagar sobre la presencia de L. flavicauda para delimitar el límite de su distribución hacia el noreste.

Tabla 1. Sitios de muestreo por sectores en las regiones de San Martin y Huánuco. Los números en paréntesis están referidos a la ubicación de los sitios de muestreo en la Figura 1.

\begin{tabular}{|c|c|c|c|c|c|}
\hline Región & Sector & Sitios de muestreo & $\begin{array}{l}\text { Coordenadas } \\
\text { geográficas }\end{array}$ & $\begin{array}{l}\text { Altitud } \\
\text { (m.s.n.m) }\end{array}$ & $\begin{array}{l}\text { Nivel de } \\
\text { perturbación } \\
\text { humana }\end{array}$ \\
\hline \multirow{3}{*}{ San Martín } & Microcuenca del río Mishollo & Hoja Grande (1) & $8^{\circ} 09^{\prime} 42^{\prime \prime} / 76^{\circ} 52^{\prime} 25^{\prime \prime}$ & 1833 & Alto \\
\hline & Microcuenca del río Tocache & Shunté (2) & $8^{\circ} 26^{\prime} 29^{\prime \prime} / 76^{\circ} 49^{\prime} 54^{\prime \prime}$ & 1843 & Alto \\
\hline & Microcuenca del río Crisnejas & Tingo de Uchiza (3) & $8^{\circ} 28^{\prime} 37^{\prime \prime} / 76^{\circ} 34^{\prime} 54^{\prime \prime}$ & 1061 & Alto \\
\hline \multirow{3}{*}{ Huánuco } & Microcuenca del río Chontayacu & San Antonio (4) & $8^{\circ} 36^{\prime} 56^{\prime \prime} / 76^{\circ} 30^{\prime} 01^{\prime \prime}$ & 1633 & Muy alto \\
\hline & Microcuenca del río Monzón & Chapákara (5) & $9^{\circ} 14^{\prime} 31^{\prime \prime} / 76^{\circ} 33^{\prime} 39^{\prime \prime}$ & 1575 & Muy alto \\
\hline & Ríos Huallaga - Pozuzo & Libertad (6) & $9^{\circ} 44^{\prime} 18^{\prime \prime} / 75^{\circ} 40^{\prime} 17^{\prime \prime}$ & 1673 & Alto \\
\hline
\end{tabular}




\section{Material y métodos}

Censos.- Para este propósito fueron abiertos entre tres a cinco trochas por sitio de muestreo, cuyas longitudes, dependiendo de las condiciones del relieve, variaron desde 2.5 a $3.0 \mathrm{~km}$. En ocasiones también recurrimos al uso de trochas de los extractores de productos forestales, agricultores y cazadores. Cada una de las trochas fue recorrida de 2 a 3 veces en forma intercalada. Los censos fueron de ida y vuelta, desde las 07:00 a 16:00 h (diurno) y 18:30 a 20:30 h (nocturno). Los censos nocturnos fueron conducidos en San Antonio, Tingo de Uchiza y Shunté y luego suspendidos para evitar accidentes por el cansancio y fatiga muscular, consecuencia de los recorridos diurnos. Dos equipos conformados por un investigador y un asistente de campo se movilizaron simultáneamente a lo largo de las trochas a una velocidad promedio de $0.5 \mathrm{~km} /$ hora. Cada vez que un grupo de primates fue avistado se procedió a anotar en la libreta de campo los siguientes datos: hora de contacto, especie, tamaño de grupo (cuando fue posible el conteo completo), distancia perpendicular del primer individuo observado al transecto, altura (en el estrato vertical del bosque), actividad al momento de su detección, presencia de juveniles e infantes y tipo de vegetación circundante. Adicionalmente se anotaron datos relacionados al nivel de perturbación del bosque y las actividades antrópicas que se desarrollan. En total, se recorrieron $928 \mathrm{~km}$ equivalentes a 1617 horas de censo. De ellos, $426 \mathrm{~km}$ (entre diurno y nocturno) correspondió a los bosques montanos de San Martín y el resto a Huánuco (Tabla 2).

Entrevistas.- las preguntas estuvieron enfocadas a la obtención de información acerca de las especies que habitan en el entorno de su comunidad, recursos alimenticios (nombre común de las plantas), épocas de nacimiento y migraciones, propósito y especies cazadas, proyectos de construcción de carreteras e hidroeléctricas, existencia de concesiones forestales, mineras y de hidrocarburos, entre otros. Por cada sitio de muestreo fueron entrevistadas dos personas, quienes normalmente participaron como guías de campo.

Análisis de datos.- El número de grupos de primates registrados para cada especie durante los censos (menores a ocho muestras) no fueron suficientes para calcular la densidad poblacional. Por tal motivo optamos por estimar únicamente la abundancia relativa; es decir, el número de individuos avistados/10 km de longitud recorrida. Para este propósito, primero fue estimado el número de grupos $/ 10 \mathrm{~km}$, mediante una regla de tres simple, luego este resultado fue multiplicado por el tamaño promedio de grupo de cada especie. La abundancia relativa estimada está basada sobre el total de longitud recorrida en cada región, excepto para $A$. chamek, cuya estimación fue en base a $373 \mathrm{~km}$, considerando que en el lado occidental del río Huallaga el límite de su distribución al norte sería el río Monzón. Se excluyen del análisis a Lagothrix lagothricha tschudii y Aotus cf. miconax por no contar con suficientes grupos registrados.

El tamaño promedio de grupo fue determinado de grupos con conteo completo. Para este propósito, cada vez que hubo contacto con primates procuramos contar a todos los individuos del grupo y cuando fue posible también a los adultos (hembras y machos), juveniles e infantes. Los infantes sin locomoción propia no fueron considerados para determinar el tamaño promedio de grupo.

\section{Resultados}

Especies y grupos registrados.- En total fueron avistados 49 grupos pertenecientes a 7 especies (Tabla 3). De ellos, la mayoría de grupos se observaron en Shunté (Región San Martin) y Libertad (Región Huánuco) con 12 y 11 respectivamente. Lagothrix flavicauda fue la más observada (Fig.2) con 14 grupos, seguidos por Cebus yuracus (Fig. 3) y Sapajus macrocephalus con 11 y 10 , respectivamente (Tabla 3). Ateles chamek fue avistado en Chapákara y Libertad y el único grupo de L. lagothricha tschudii en Libertad. De otro lado, los escasos registros de Alouatta seniculus (Fig.4) mayormente ocurrieron en Chapákara y Hoja Grande; en el resto, con excepción de Libertad no fue observado, pero se escucharon algunas vocalizaciones.

Tamaño de grupo y rango de variación.- Grupos más grandes se registraron en $L$. flavicauda y $C$. yuracus, cuyo tamaño promedio y rango de variación resultaron similares entre los observados en San Martín y Huánuco (Tabla 4). Lo contrario ocurrió en $A$. seniculus, en particular en Huánuco con un tamaño promedio de 3 y rango de 2 a 5 individuos. En S. macrocephalus, el tamaño promedio de grupo en San Martín fue 5.3 individuos, mientras que para los que habitan en Huánuco resultó imposible determinarlo. En San Antonio, grupos pequeños de S. macrocephalus y C. yuracus fueron observados; sin embargo, no fue posible un conteo completo por su conducta huidiza propio de aquellos que están sometidos a la caza o son repelidos con armas de fuego. Lo opuesto ocurrió en Shunté y Libertad, donde los grupos observados, en particular de L. flavicauda se caracter-

Tabla 2. Distancia recorrida y horas censadas por sitio de muestreo entre las Regiones de San Martín y Huánuco.

\begin{tabular}{|c|c|c|c|c|c|}
\hline \multirow{2}{*}{ Sector } & \multirow{2}{*}{$\begin{array}{l}\text { Sitio de } \\
\text { muestreo }\end{array}$} & \multicolumn{2}{|c|}{ Longitud recorrida por sitio de muestreo $(\mathrm{km})$} & \multirow{2}{*}{$\begin{array}{l}\text { Longitud total } \\
\text { recorrida }(\mathbf{k m})\end{array}$} & \multirow{2}{*}{$\begin{array}{l}\text { Esfuerzo total } \\
\text { (horas) }\end{array}$} \\
\hline & & Diurno & Nocturno & & \\
\hline Microcuenca del río Mishollo & Hoja Grande & 138 & & 138 & 230 \\
\hline Microcuenca del río Tocache & Shunté & 168 & 14 & 182 & 303 \\
\hline Microcuenca del río Crisnejas & Tingo de Uchiza & 95 & 11 & 106 & 176 \\
\hline Microcuenca del río Chontayacu & San Antonio & 109 & 20 & 129 & 215 \\
\hline Microcuenca del río Monzón & Chapákara & 160 & & 160 & 267 \\
\hline \multirow[t]{2}{*}{ Ríos Huallaga - Pozuzo } & Libertad & 213 & & 213 & 426 \\
\hline & Total & 883 & 45 & 928 & 1617 \\
\hline
\end{tabular}




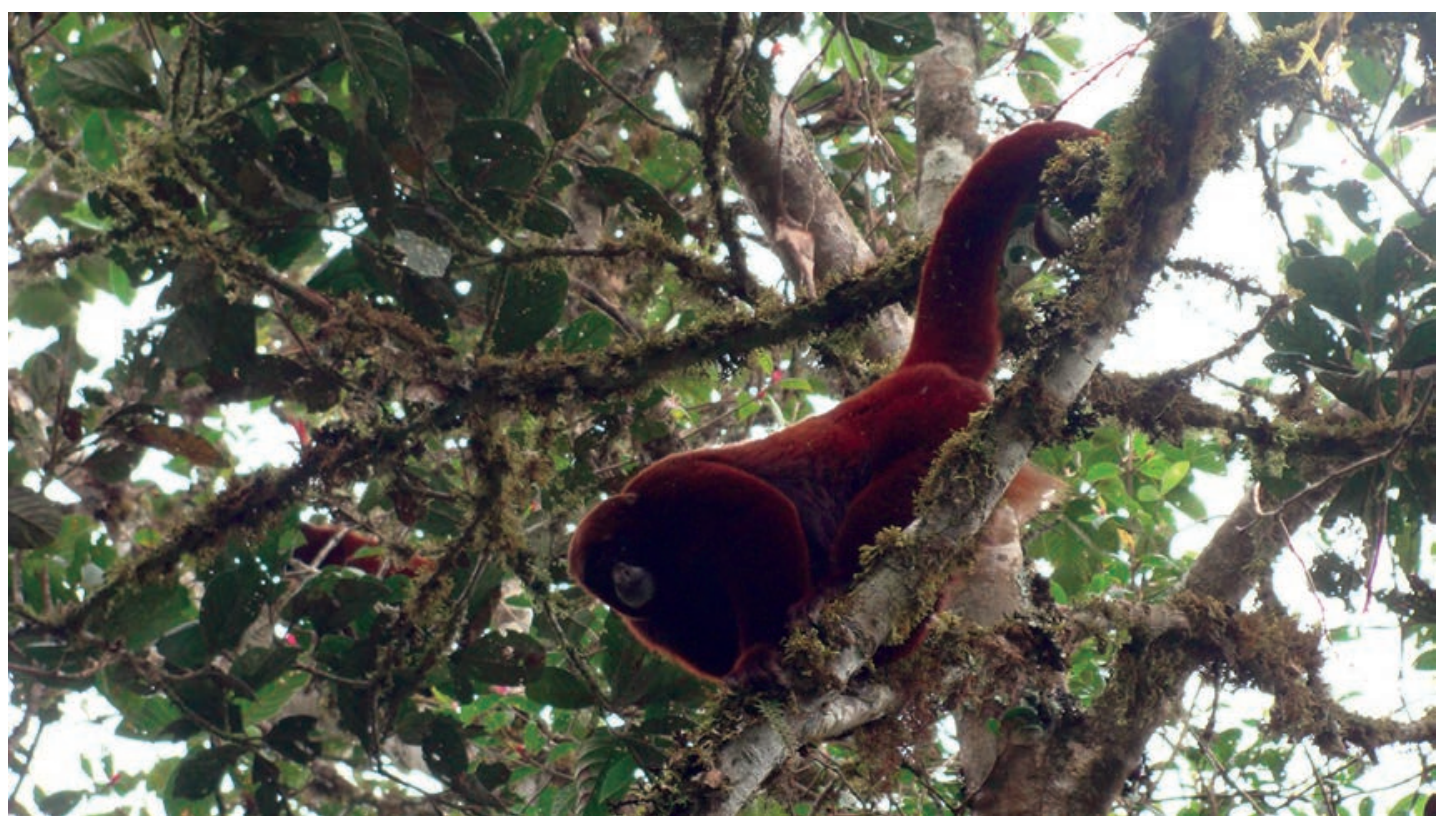

Figura 2. Macho adulto de Lagothrix flavicauda observado en Hoja Grande, San Martín.

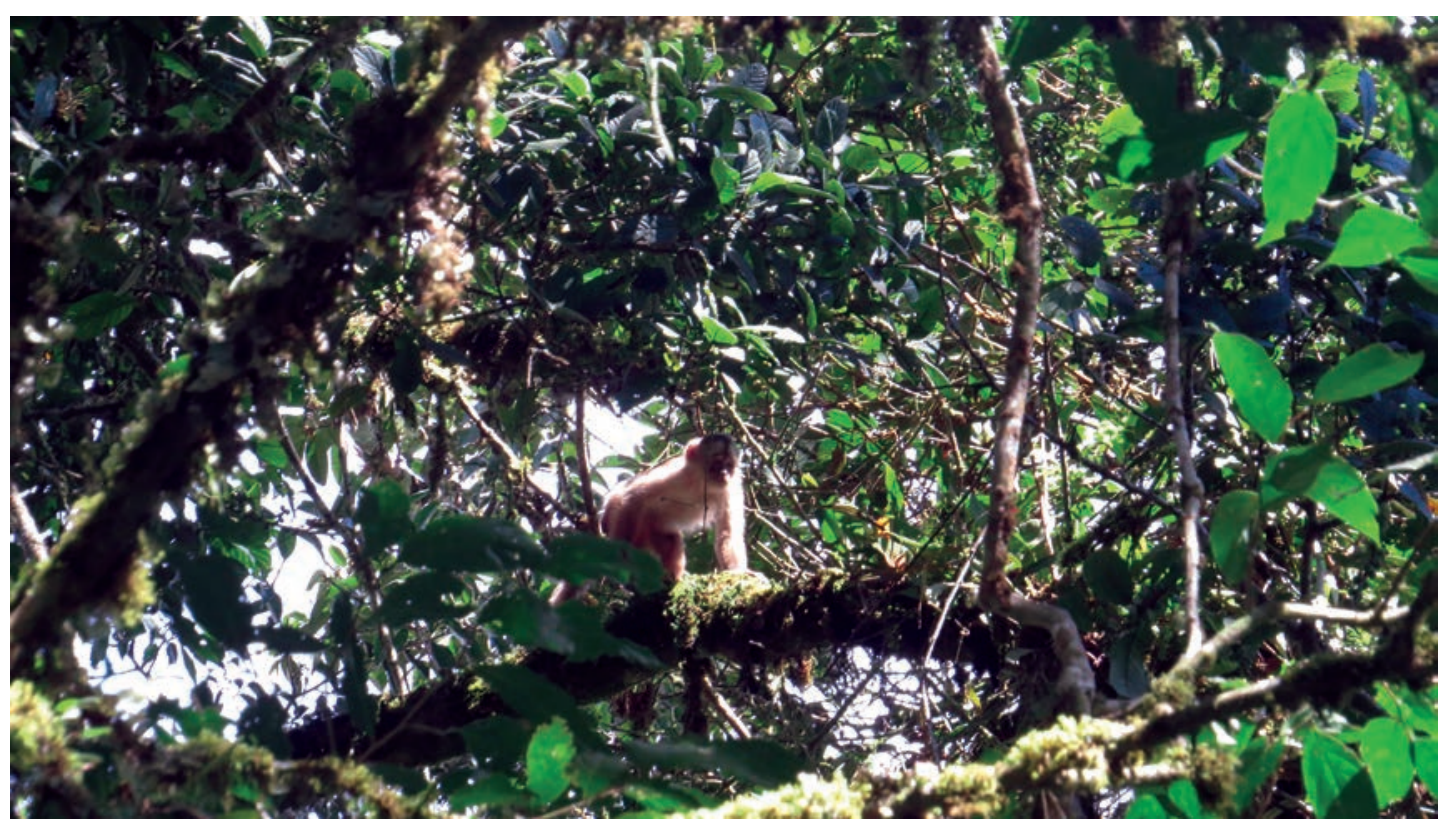

Figura 3. Macho adulto de Cebus yuracus observado en Hoja Grande, San Martín.

Tabla 3. Especies y grupos registrados en los sitios de muestreo de San Martín y Huánuco.

\begin{tabular}{|c|c|c|c|c|c|c|c|}
\hline \multirow{2}{*}{ Especies } & \multicolumn{3}{|c|}{ San Martín } & \multicolumn{3}{|c|}{ Huánuco } & \multirow{2}{*}{ Total } \\
\hline & Hoja Grande & Shunté & Tingo de Uchiza & San Antonio & Chapákara & Libertad & \\
\hline Lagothrix flavicauda & 2 & 4 & 2 & 1 & & 5 & 14 \\
\hline Ateles chamek & & & & & 1 & 1 & 2 \\
\hline Lagothrix l. tschuddi & & & & & & 1 & 1 \\
\hline Alouatta seniculus & 3 & & & & 3 & 1 & 7 \\
\hline Cebus yuracus & 2 & 2 & 2 & 1 & 3 & 1 & 11 \\
\hline Sapajus macrocephalus & 2 & 4 & 1 & 2 & & 1 & 10 \\
\hline Aotus cf. miconax & & 2 & 1 & & & 1 & 4 \\
\hline Total & 9 & 12 & 6 & 3 & 7 & 11 & 49 \\
\hline
\end{tabular}




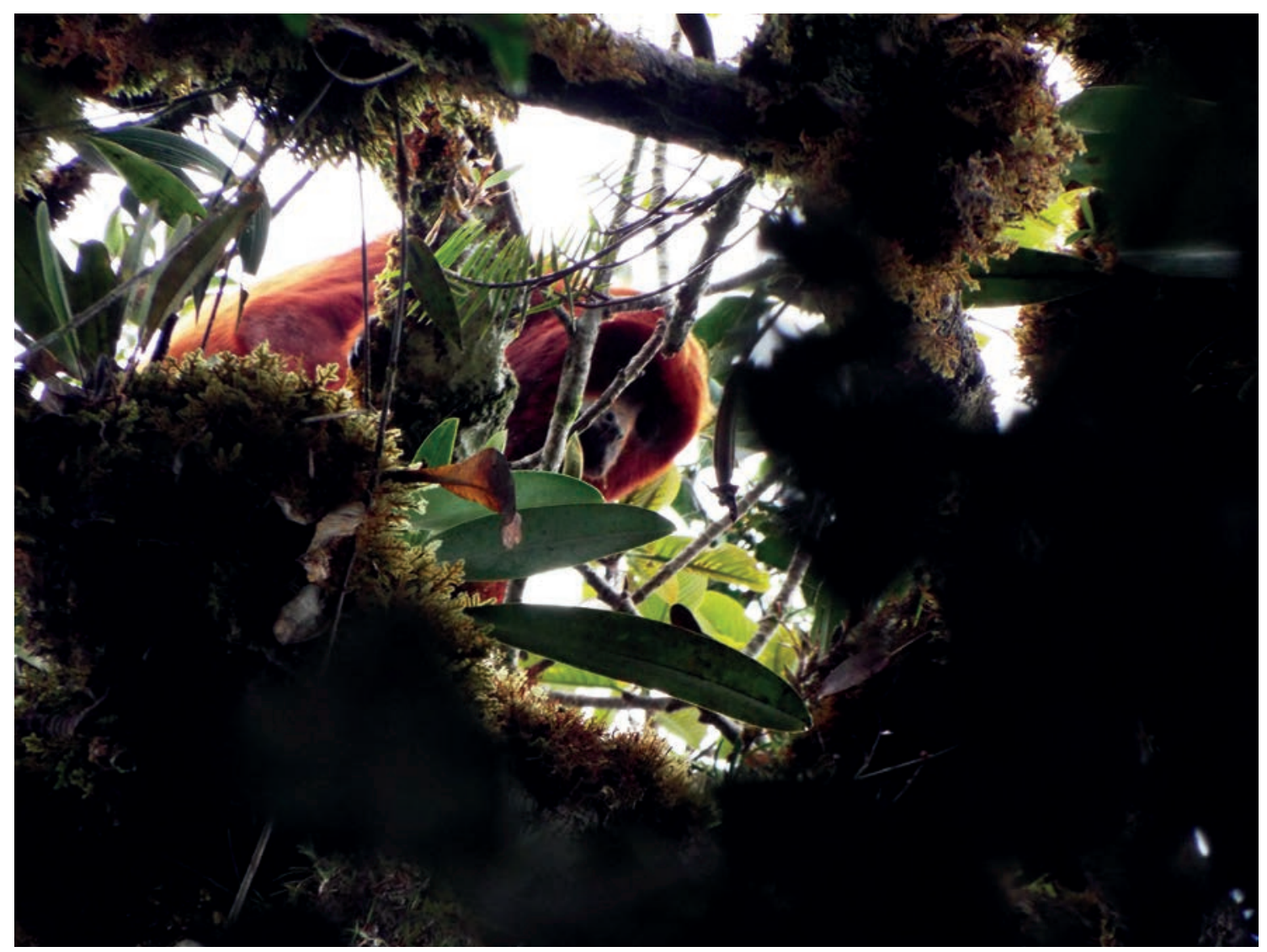

Figura 4. Ejemplar adulto de Alouatta seniculus observado en Chapákara, Región Huánuco.

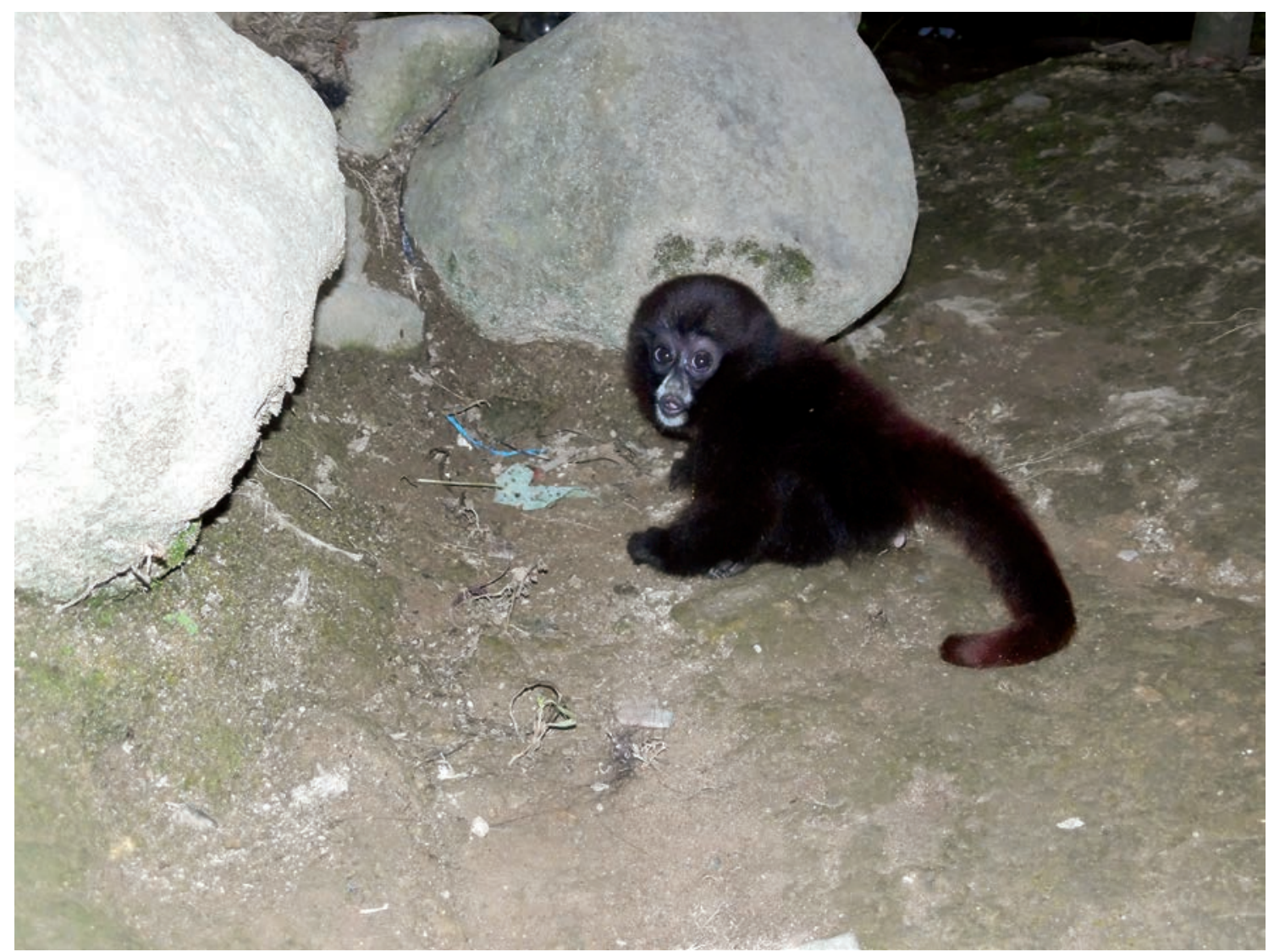

Figura 5. Infante de Lagothrix flavicauda destinado a la venta para mascota en Shunté, San Martín. 
izaron por su tolerancia a la presencia humana, lo que permitió un conteo completo en algunos grupos. En el caso de Aotus cf. miconax, el tamańo promedio para los grupos observados en San Martín fue calculado en 3.3 individuos, promedio que está dentro del estimado para otras áreas, considerando que las especies de este género viven en parejas y pequeños grupos familiares, de modo que salvo excepciones, no es común encontrar grupos mayores a cinco individuos.

Abundancia relativa.- En términos generales fue mayor para las especies que habitan en San Martín (Tabla 5). Comparando entre especies, la mayor abundancia correspondió a $L$. flavicauda tanto en San Martín como en Huánuco (2.4 y 1.6 individuos $/ 10 \mathrm{~km}$, respectivamente), mientras que la más baja fue para A. seniculus, en particular en Huánuco con apenas 0.24 individuos/ $10 \mathrm{~km}$.

Ampliación de distribución de L. flavicauda-. Con el avistamiento de grupos entre Oso Mayo y Libertad, el área de distribución de esta especie se amplía hacia el lado oriental, desde muy cerca del río Huallaga hasta el río Pozuzo y aproximadamente desde el río Tulumayo en el norte hasta el límite con la Región Pasco en el sur. Su distribución podría incluso extenderse hasta los bosques montanos de las regiones de Ucayali y Pasco cerca al límite con la Región Huánuco.

Ampliación de distribución de $\boldsymbol{A}$. chamek-. El registro de un grupo en Chapákara correspondiente al Alto Monzón nos permite sostener que esta especie también está presente en la margen izquierda del río Huallaga, cuyo límite hacia el norte sería el Río Monzón. No obstante, con excepción del Parque
Nacional de Tingo María y los bosques entre los ríos Monzón y Yanamayo, en el resto de su área estaría localmente extinta.

Amenazas y situación actual.- En los sitios de muestreo de San Martín y Huánuco, L. flavicauda y los otros primates se encuentran habitando en parches de bosque residual y primario, este último presente en las laderas empinadas y de difícil acceso. En San Martín, estos bosques se encuentran en el curso superior y cabeceras de las microcuencas de los ríos Tocache, Crisnejas y Mishollo, en donde con excepción de la última, están expuestos a la deforestación para la agricultura, ganadería y extracción de recursos forestales, con la consecuente pérdida de hábitats. A ello se suma la caza que de acuerdo a los entrevistados es ocasional y orientado hacia el consumo de subsistencia y la obtención de infantes para la venta como mascotas (Fig. 5). No obstante, en estos últimos años, estas actividades habrían disminuido en intensidad, al menos así nos manifestaron algunos pobladores de las comunidades de Shunté y Tingo de Uchiza, quienes incluso mostraron su interés por contar con áreas de conservación y para los fines de ecoturismo. Esta decisión por parte de los pobladores al parecer está dando sus resultados, en particular en Shunté, donde registramos 12 grupos de primates, de los cuales 4 fueron de L. flavicauda. En lo que corresponde a las cabeceras de la microcuenca del río Mishollo, por ahora la deforestación para la agricultura y ganadería es moderada, pero la extracción de recursos forestales y la caza va en aumento, al menos así lo percibimos durante nuestra permanencia al interior del bosque y está afectando principalmente a los mamíferos considerados de tamaño grande, entre ellos a L. flavicauda, especie que fue observada en solo dos ocasiones.

Tabla 4. Tamaño de grupo y rango de variación de primates observados en bosques de San Martin y Huánuco.

\begin{tabular}{|c|c|c|c|c|c|}
\hline \multirow{2}{*}{ Especies } & \multicolumn{2}{|l|}{ San Martín } & \multicolumn{2}{|l|}{ Huánuco } & \multirow{2}{*}{$\begin{array}{c}\text { Rango de } \\
\text { variación en } \\
\text { otras áreas }\end{array}$} \\
\hline & Tamaño promedio de grupo & Rango & Tamaño promedio de grupo & Rango & \\
\hline Lagothrix flavicauda & $12 \pm 3.6(\mathrm{~N}=4)$ & $8-15$ & $13.5 \pm 2.2(\mathrm{~N}=2)$ & $12-15$ & $3-19^{a}$ \\
\hline Ateles chamek & & & $6 \pm 3(\mathrm{~N}=2)$ & $3-9$ & $8-17^{b}$ \\
\hline Alouatta seniculus & $4 \pm 1(\mathrm{~N}=3)$ & $3-5$ & $3 \pm 2.4(\mathrm{~N}=3)$ & $2-5$ & $4-11^{\mathrm{a}}$ \\
\hline Cebus yuracus & $9.5 \pm 1(\mathrm{~N}=2)$ & $9-13$ & $12.5 \pm 3.6(\mathrm{~N}=2)$ & $10-15$ & $6-13^{b}$ \\
\hline Sapajus macrocephalus & $5.3 \pm 2.1(\mathrm{~N}=3)$ & 3- 9 & & $>5$ & $7-11^{b}$ \\
\hline Aotus cf. miconax & $3.3 \pm 1(\mathrm{~N}=3)$ & $3-4$ & & $>1$ & \\
\hline
\end{tabular}

a Chontayacu-Miraflores: Aquino et al. (2015a).

b Urubamba y Tambo: Aquino et al.(2013).

Tabla 5. Abundancia relativa de primates estimada para los bosques montanos de San Martín y Huánuco.

\begin{tabular}{|c|c|c|c|c|c|c|c|}
\hline \multirow{2}{*}{ Especies } & \multicolumn{2}{|c|}{ Longitud total recorrida $(\mathrm{km})$} & \multicolumn{2}{|c|}{ Grupos observados } & \multicolumn{3}{|c|}{ Individuos/ $10 \mathrm{~km}$} \\
\hline & San Martín & Huánuco & San Martín & Huánuco & San Martín & Huánuco & Otras áreas* \\
\hline Lagothrix flavicauda & 401 & 482 & 8 & 6 & 2.4 & 1.6 & $0.5^{\mathrm{a}}$ \\
\hline Ateles chamek & & 373 & & 2 & & 0.3 & $0.36^{\mathrm{a}}$ \\
\hline Alouatta seniculus & 401 & 482 & 3 & 4 & 0.3 & 0.2 & $0.24^{\mathrm{a}}$ \\
\hline Cebus yuracus & 401 & 482 & 6 & 5 & 1.4 & 1.2 & $0.08^{\mathrm{a}}$ \\
\hline Sapajus macrocephalus & 401 & 482 & 7 & 3 & 0.9 & 0.3 & $0.1^{\mathrm{a}}$ \\
\hline
\end{tabular}

*Las cifras están referidos a grupos $/ 10 \mathrm{~km}$.

a Chontayacu-Miraflores: Aquino et al. (2015a). 
En Huánuco, con excepción de Oso Mayo y Libertad correspondiente al sector Huallaga - Pozuzo, los resultados obtenidos para los otros sitios de muestreo indican escasa o nula presencia de L. flavicauda y de otros primates y se debe entre otros factores a la pérdida de hábitats por la intensa deforestación para la agricultura, ganadería y extracción de madera, pero también a la caza para la obtención de la piel y de infantes que son ofertados para artesanía y mascota, respectivamente. Otra de las actividades que contribuyen a la perturbación del bosque es la extracción de resina de Clusia sp. (Familia Clusiaceae) para incienso con fines comerciales, cuyo continuo sangrado según los entrevistados ocasiona la muerte de los árboles más jóvenes. Esta actividad contribuye al incremento de la caza tanto en San Antonio como en Chapákara por las numerosas trochas que se han abierto para la búsqueda y localización de los árboles de Clusia sp., los mismos que son utilizados para la caza. Entre las especies más afectadas por las actividades mencionadas figuran los atélidos, en particular L. flavicauda, prácticamente ausente en San Antonio y Chapákara, aun cuando los entrevistados nos manifestaron la presencia de este y otros primates en determinadas épocas del año coincidente con la producción de frutos.

Entre Oso Mayo y Libertad, la principal amenaza es la deforestación para los fines agrícolas y ganaderas, habiendo afectado al bosque en una amplitud de aproximadamente $3 \mathrm{~km}$, es decir, $1.5 \mathrm{~km}$ por cada lado de la herradura que conecta a los ríos Huallaga y Pozuzo. Sin embargo, aún existe bosque residual esencialmente primario en buen estado de conservación, en particular hacia el norte de la herradura donde estaría concentrada gran parte de la población de L. flavicauda y de otros primates. En este sector, la caza es muy esporádica y al parecer no afecta a L. flavicauda, porque está orientado hacia los primates y otros mamíferos que ocasionan pérdidas económicas como $S$. macrocephalus y $C$. yuracus, quienes incursionan principalmente a los maizales para alimentarse del maíz inmaduro más conocido como "choclo", por lo que son repelidos con armas de fuego para ahuyentarlos, aunque algunas veces son impactados por las municiones. Sin embargo, el buen estado de la población de L. flavicauda aquí presente podría cambiar drásticamente en el mediano plazo porque está previsto continuar con la construcción de la carretera para conectar las cuencas de los ríos Huallaga y Pozuzo, facilitando el acceso de más colonos lo que intensificará la deforestación para la agricultura, ganadería y comercialización de madera con la consecuente pérdida de hábitats.

\section{Discusión}

La diversidad de primates registrada en este estudio es similar a la reportada para Chontayacu-Miraflores (Aquino et al. 2015a) y Alto Azul (Aquino et al. 2016a). Es mayor al registrado por Shanee et al. (2013b) para los bosques pre montano y montano de Monzón y Ajenco, ambos en la Región Huánuco y resultó mayor a los registros para el bosque montano de Cajamarca (Aquino et al. 2014). De las siete especies, cinco fueron observadas en bosques de San Martín y todas en Huánuco, entre ellas A. chamek y L. lagothricha tschudii. Estas dos especies no fueron registradas en San Martín, porque de acuerdo con Aquino y Encarnación (1994) y Aquino et al. (2015b) no forman parte de su área de distribución.

El rango y tamańo promedio de grupos de L. flavicauda observados en San Martín y Huánuco son similares a los reportados para el Parque Nacional Río Abiseo (Leo Luna 1982), La
Esperanza (Shanee \& Shanee 2011) y Chontayacu-Miraflores (Aquino et al. 2015a). El tamaño y rango de grupos registrados en este estudio resulta menor al reportado por DeLuycker (2007) para el Bosque de Protección Alto Mayo, quien observó grupos de 10 a más de 25 individuos; pero es mayor al registrado por Cornejo (2008) en el Área de Conservación Privada Abra Patricia-Alto Nieva, cuyo tamaño promedio de grupo fue estimado en 5.53 y rango de 2 a 7 individuos. Con referencia a las otras especies, el tamaño de grupo, particularmente de $A$. chamek y $A$. seniculus resulta menor al registrado en el interfluvio entre los ríos Urubamba-Tambo (Aquino et al. 2013) y Miraflores (Aquino et al. 2015a), sitios de muestreo donde fueron observados grupos de hasta 15 individuos para la primera de las citadas y nueve individuos para la segunda especie.

La abundancia relativa estimada para L. flavicauda en San Martín es mayor a la de Huánuco (este estudio), pero resulta menor a lo reportado para Yanajanca-Cocalito en Huánuco (Aquino et al., 2015a, 2015c), quienes estimaron la abundancia de L. flavicauda en 0.5 grupos $/ 10 \mathrm{~km}$, equivalente a 6.8 individuos $/ 10 \mathrm{~km}$. Estas diferencias tienen mucho que ver con la calidad y extensión del bosque, mientras que algunos se encuentran en buen estado de conservación, otros en cambio muestran un perfil de alta perturbación humana como es el caso de San Antonio y Chapákara. La abundancia relativa estimada para el resto de especies, tanto en San Martín como en Huánuco son menores a las estimadas para el interfluvio entre los ríos Urubamba-Tambo (Aquino et al. 2013), bosques premontano y montano de Cajamarca (Aquino et al. 2014) y Chontayacu-Miraflores (Aquino et al. 2015a), sitios donde el bosque residual esencialmente primario todavía se encuentra en buen estado de conservación.

El límite de distribución de L. flavicauda hacia el sur determinado en este estudio contrasta con Shanee (2011) y con Aquino et al. (2015a) quienes mencionan al río Magdalena y a los bosques montanos de la margen izquierda del río Huallaga, respectivamente; pero coincide con Aquino et al. (2016b), quienes consideran hasta el límite con la Región Pasco. Es muy probable que su distribución podría incluso ampliarse hasta los bosques montanos de Pasco y Ucayali, por lo que sería de mucho interés realizar exploraciones para salir de dudas. Por otro lado, la distribución de $A$. chamek en Huánuco determinada en este estudio, sobrepasa el límite propuesto por Aquino y Encarnación (1994) y Aquino et al. (2015b), quienes consideran únicamente el lado oriental del río Huallaga. En ambos casos no tomaron en cuenta la existencia de esta especie en el Parque Nacional de Tingo María (SERNANP 2015). El registro de un grupo en bosque montano aledaño al centro poblado de Chapákara y la ausencia de registros al sur del río Monzón en estudios que nos antecedieron (Aquino et al.2015a, 2016a) nos indicaría que en el lado occidental del Huallaga el límite de su distribución hacia el norte sería el río Monzón. No obstante, se requiere realizar muestreos en otros sitios, en particular entre los ríos Monzón y Magdalena para validar o descartar el límite aquí propuesto.

En el área de estudio, la predominancia de grupos pequeños y baja abundancia de primates para la mayoría de las especies estaría asociada principalmente con la alteración y pérdida de hábitats por la deforestación con fines agrícolas, ganaderas y extracción de madera y otros recursos forestales; pero también tiene mucho que ver con la caza, en particular de $S$. macrocephalus 
y C. yuracus, considerados como plagas por los agricultores. De acuerdo con Estrada et al. (2017), ambas actividades son consideradas por la IUCN como las principales amenazas para los primates que habitan en el mundo y son los responsables para que el 36\% de las especies que habitan en el neotrópico estén amenazadas y cuyas poblaciones hayan declinado en un $63 \%$. Se sobreentiende que en este porcentaje están incluidas gran parte de las especies, en particular L. flavicauda.

De las actividades mencionadas, la pérdida de hábitats por la intensa deforestación para los fines antes mencionados, es sin duda el problema más crítico para los primates y otros componentes de la fauna silvestre, coincidiendo así con las sostenidas por Shanee (2011) para los primates que habitan los bosques montanos de Amazonas, La Libertad, Huánuco y San Martín. No obstante, todavía existen fragmentos relativamente extensos de bosques residuales esencialmente primarios en las cabeceras de los ríos Tocache y Mishollo en San Martin y entre Oso Mayo y Libertad en Huánuco. En estos bosques aún se encuentran poblaciones de L. flavicauda y otros primates similares a los reportados para los bosques entre las microcuencas de los ríos Chontayacu y Yanajanca (Aquino et al. 2015a). Estos bosques deberían ser tomados en cuenta para su conservación y el ecoturismo, muy en particular de Shunté y entre Oso Mayo y Libertad, donde fueron observados la mayoría de los grupos de L. flavicauda, lo que indica que estos sitios de muestreo están en mejores condiciones que el resto, aun cuando entre Oso Mayo y Libertad hubo escasa presencia de otras especies de primates, pero alta diversidad de otros componentes de la fauna silvestre, en particular aves.

En referencia a la caza, la mayor presión posiblemente es ejercida en bosques aledaños a Hoja Grande, San Antonio y Chapákara, donde existen ciertas familias de descendencia amazónica que por tradición recurren al uso de la fauna silvestre para proveerse de proteína animal. Por otro lado, en Shunté, Tingo de Uchiza y Libertad, la caza al parecer es esporádica porque la mayoría de la gente asentada en estas comunidades es de descendencia andina. Los andinos salvo excepciones, generalmente no utilizan a la fauna silvestre para el consumo de subsistencia, limitando sus actividades a la agricultura y ganadería, por lo que no es extraño la presencia de L. flavicauda, A. chamek y otros primates incluso en parches de bosque residual cercanos a los campos de cultivo y pastizales. En estos sitios de muestreo, la caza según manifestaron algunos pobladores está orientada mayormente al control de poblaciones de ciertas especies consideradas plagas para la agricultura entre los que se encuentran S. macrocephalus y C. yuracus, más conocidos como "chocleros" o "maiceros". El ingreso a los campos de cultivo podría estar relacionado con aquellos grupos que habitan en bosques fuertemente degradados o en fragmentos de bosques relativamente pequeños y coincidentes con la época de escasez de recursos alimenticios, tal como fue demostrado en Cerro Preto y Boa Esperança en el estado de Rio Grande do Sul (Rocha \& Fortes, 2015) y al sureste de Brasil (Freitas et al. 2008), respectivamente. La diferencia está en el trato a estos animales, mientras que en el área de estudio se opta por la caza con armas de fuego para disminuir las poblaciones, en Brasil reconocen la importancia de estos primates en el bosque, por lo que para ahuyentarlos, la mayoría recurren a la emisión de sonidos fuertes o al uso de perros, pero no los matan.

\section{Agradecimientos}

Nuestro agradecimiento a The Mohamed bin Zayed Species Conservation Fund por el apoyo económico que nos ha permitido continuar con el inventario y evaluación de los primates en bosques montanos de las regiones de San Martin y Huánuco (Proyecto \# 152510817). También agradecemos a la Universidad Nacional Mayor de San Marcos por el apoyo económico para la adquisición de materiales de campo (código estudio: 161001211). Finalmente, hacemos extensivo nuestra reconocimiento a los guías de campo de las comunidades de Shunté, Tingo de Uchiza, San Antonio, Chapákara, Bellavista y Libertad por su apoyo incondicional durante las actividades de campo.

\section{Literatura citada}

Aquino R. \& F. Encarnación. 1994. Primates of Peru/ Los Primates del Perú. Primate Report 40: 1-130.

Aquino R. \& C. Ramos. 2010. Fauna de la Selva de Huánuco. Informe Final. Proyecto Meso Zonificación Ecológica y Económica para el desarrollo sostenible de la selva de Huánuco. Convenio entre el Instituto de Investigaciones de la Amazonía Peruana (IIAP) y la Comisión Nacional para el Desarrollo y Vida (DEVIDA). Huánuco. 57 pp.

Aquino R., F. Cornejo \& E.W. Heymann. 2013. Primate abundance and habitat preferences on the lower Urubamba and Tambo rivers, central-eastern Peruvian Amazonia. Primates 54:377383. http://dx.doi.org/10.1007/s10329-013-0357-3

Aquino R., E. Charpentier, G. García, et al. 2014. Reconocimiento de primates y amenazas para su supervivencia en bosques premontano y montano de la Región Cajamarca, Perú. Neotropical Primates 21(2):171-176. http://dx.doi. org/10.1896/044.021.0202

Aquino R., R. Zárate, L. López, et al. 2015a. Current status and threats to Lagothrix flavicauda and other primates in montane forest of the Región Huánuco. Primate Conservation 29: 1-11. http://dx.doi.org/10.1896/052.029.0111

Aquino R., F. Cornejo, L. Cortés, et al. 2015b. Primates del Perú: Guía de identificación de bolsillo. Conservation International, Arlington, Virginia, USA.

Aquino, R., L. López, G. García \& E. Charpentier. 2015c. Inventario y evaluación de primates y amenazas para sus poblaciones en bosque montano de la Región Huánuco, Perú. Ciencia Amazónica (Iquitos) 5(1): 61-69.

Aquino R., G. García \& E. Charpentier. 2016a. Distribution and current status of the Peruvian yellow-tailed woolly monkey (Lagothrix flavicauda) in montane forests of the Región Huánuco, Perú. Primate Conservation 30: 31-37. http:// dx.doi.org/10.1896/052.029.0111

Aquino R., E. Charpentier, G. García, et al. 2016b. First Record of Lagothrix flavicauda on the eastern side of the Río Huallaga: An expansion of its known geographic distribution. Primate Conservation 30: 15-20.

Butchart S.H.M., R. Barnes, C.W.N. Davies, et al. 1995. Observation of two threatened primates in the Peruvian Andes. Primate Conservation 16: 15-19.

Cornejo F.M. 2008. Aspects of the ecology and behavior of the yellowtailed woolly monkey Oreonax flavicauda Humboldt 1802. XXII Congress of the International Primatological Society, Edinburgh, UK, 3-8 August 2008. Primate Eye (96) Special Issue: 151.

Cornejo F., R. Aquino \& C. Jiménez. 2008. Notes on the natural history, distribution and conservation status of the Andean night monkey, Aotus miconax, Thomas 1927. Primate Conservation 23: 1-4. http://dx.doi.org/10.1896/052.023.0101

De Luycker A.M. 2007. Notes on the yellow-tailed woolly monkey (Oreonax flavicauda) and its status in the Protected Forest of Alto Mayo, Northern Peru. Primate Conservation 22: 41-47. http://dx.doi.org/10.1896/052.022.0102

Estrada A., P.A. Garber, A.B. Rylands, et al. 2017. Impending extinction crisis of the world's primates: Why primates matter. Science Advances 3:e1600946. http://dx.doi.org/10.1126/ sciadv. 1600946. 
Freitas C.H., E.Z. Setz, A.R. Araujo \& N. Gobbi. 2008, Agricultural crops in the diet of bearded capuchin monkeys, Cebus libidinosus Spix (Primates: Cebidae), in forest fragments in southeast Brazil. Revista Brasileira de Zoologia 25:32-39. http://dx.doi.org/10.1590/S0101-81752008000100006

Leo Luna M. 1980. First field study of the yellow-tailed woolly monkey. Oryx 15: 386-389.

Leo Luna M. 1982. Estudio preliminar sobre la biología y ecología del mono choro de cola amarilla Lagothrix flavicauda (Humboldt 1812). Tesis, Título Profesional de Bióloga, Facultad de Ciencias, Universidad Nacional Agraria La Molina, Lima. 169 pp.

Leo Luna, M. 1987. Primate conservation in Peru: a case study of the yellow-tailed woolly monkey. Primate Conservation 8: 122-123.

Mittermeier R.A., A.B. Rylands, C. Schwitzer, L.A. Taylor, F. Chiozza, E.A. Williamson (eds.). 2012. Primates in Peril: The World's 25 Most Endangered Primates 2010-2012. IUCN SSC Primate Specialist Group (PSG), International Primatological Society (IPS), Conservation International (CI) and Bristol Zoological Society, Arlington, VA.

Rocha L.C. \& V.B. Fortes. 2015. Percepçôes e atitudes de moradores rurais em relaçáo aos macacos-prego na área de influência da usina hidrelétrica Dona Francisca, Sul do Brasil. Ambiente y Sociedade 18 (4):19-34.

SERNANP (Servicio Nacional de Áreas Naturales Protegidas). 2015. Parque Nacional Tingo María, 50 ańos. Servicio Nacional de Areas Naturales Protegidas por el Estado-SERNANP. 163 pp.
Shanee S., N. Shanee \& M. Maldonado. 2008. Distribution and conservation status of the yellow-tailed woolly monkey (Oreonax flavicauda, Humboldt 1812) in Amazonas and San Martín, Peru. Neotropical Primates 14: 115-119.

Shanee S. 2011. Distribution survey and threat assessment of the yellow-tailed woolly monkey (Oreonax flavicauda; Humboldt 1812), Northeastern Peru. International Journal of Primatology 32:691-707. 691. http://dx.doi.org/10.1007/ s10764-011-9495-x

Shane S. \& N. Shane. 2011. Population density estimates of critically endangered yellow-tailed woolly monkeys (Oreonax flavicauda) at La Esperanza, Northeastern Peru. International Journal of Primatology 32: 878-888. http://dx.doi. org/10.1007/s10764-011-9507-x

Shanee S., N. Allgas-Marchena \& N. Shanee. 2013a. Preliminary observation on the behavior and ecology of the Peruvian night monkey (Aotus miconax: Primates) in a remnant cloud forest patch, north eastern Peru. Tropical Conservation Science 6 (1): 138-148. https://doi. org/10.1177/194008291300600104

Shanee S., N. Shanee \& N. Allgas-Marchena. 2013b. Primate surveys in the Marańón-Huallaga landscape, Northern Peru with notes on conservation. Primate Conservation 27: 3-11. http://dx.doi.org/10.1896/052.027.0114

Young K. \& B. León. 1999. Peru's humid eastern montane forests: An overview of their physical settings, biological diversity, human use and settlement, and conservation needs. DIVA Technical Report $\mathrm{N}^{\circ}$ 5. Denmark. http://diva.dmu.dk/1 viden/2_miljoe-tilstand/3_natur/diva/reports/diva05.pdf 